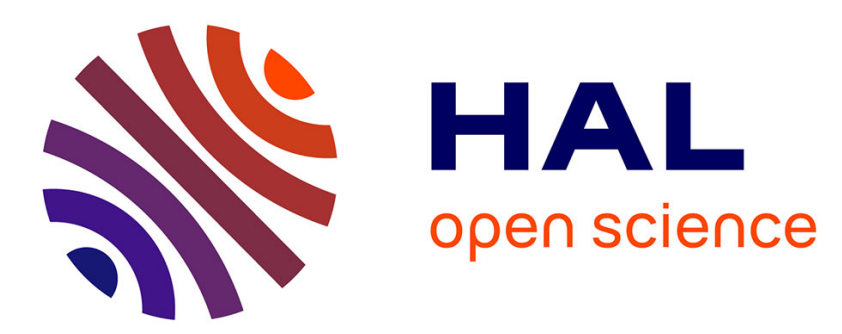

\title{
Numerical simulation of resin transfer molding using linear boundary element method
}

\author{
Fabrice Schmidt, P Lafleur, Florentin Berthet, Pierre Devos
}

\section{To cite this version:}

Fabrice Schmidt, P Lafleur, Florentin Berthet, Pierre Devos. Numerical simulation of resin transfer molding using linear boundary element method. Polymer Composites, 1999, 20 (6), pp.725-732. 10.1002/pc.10396 . hal-01716290

\section{HAL Id: hal-01716290 \\ https://hal.science/hal-01716290}

Submitted on 26 Mar 2019

HAL is a multi-disciplinary open access archive for the deposit and dissemination of scientific research documents, whether they are published or not. The documents may come from teaching and research institutions in France or abroad, or from public or private research centers.
L'archive ouverte pluridisciplinaire HAL, est destinée au dépôt et à la diffusion de documents scientifiques de niveau recherche, publiés ou non, émanant des établissements d'enseignement et de recherche français ou étrangers, des laboratoires publics ou privés. 


\title{
Numerical Simulation of Resin Transfer Molding Using Linear Boundary Element Method
}

\author{
F. M. SCHMIDT \\ Enstimac \\ Route de Teillet, 81013 Albi CT Cedex 09, France \\ P. LAFLEUR \\ CRASP, Ecole Polytechnique \\ Montreal, QC, H3C 3A7, Canada \\ F. BERTHET \\ Enstimac \\ Route de Teillet, 81013 Albi CT Cedex 09, France \\ P. DEVOS \\ Enstimac \\ Route de Teillet, 81013 Albi CT Cedex 09, France
}

\begin{abstract}
Using mass conservation and Darcy's law to describe flow through isotropic porous media leads to a Laplace equation, which may be solved numerically at each time step using the boundary element method. For anisotropic porous media in which the permeability matrix is symmetric, the problem can be solved in the same way by rotating and stretching the coordinates. The numerical model has been compared with analytical solutions and experimental data in the case of radial front flows and with finite element for a frontal injection.
\end{abstract}

\section{INTRODUCTION}

$\mathrm{T}$ The resin transfer molding (RTM) process has become popular in a variety of industries: sporting goods, defense, military, automotive, and aerospace $(1,2)$. It is widely used in the manufacturing of large components of fiber reinforced materials. Basically it is a two-step process. In the first step, a fiber preform composed of several layers of fiber mats or woven roving is produced. In the second step, the resin is injected into a mold filled with the preform. The resin enters the mold through one or several injection gates and impregnate progressively the preform with or without vacuum assistance.

The main advantage of this process in comparison with others is its adaptability. This process makes it possible to produce simultaneously a component and a material that perfectly fit the property requirements locally and globally (3). This is done by combining a variety of fiber types, levels, and architectures with a variety of cores and resins.

Because of its economic interest, this process has replaced traditional lay-up of prepregs for some applications (in particular to avoid time and labor expensive surface preparation prior to painting). Even though components are industrially produced, the filling step is still not very well understood and therefore predicted. The mold materials are seldom transparent so that the flow cannot be visualized, and it is very difficult to determine how the resin will flow and impregnate the preform. Proper design of the vent and injection port location is crucial to prevent large air pockets from being trapped during flow (if no vacuum assistance is used). The more complicated the mold, the more difficult (and also expensive) it is to adjust 
the gates' locations. This difficult task becomes an expensive one if it is done by a trial and error method. Problems can be anticipated by a proper process simulation. The numerical analysis of resin flow in the mold is a very important (technical and economical) task in designing the RTM mold and to understand the impregnation of the preform.

Several numerical methods have been developed to simulate the filling step of RTM. Cai (4) has developed a simplified filling simulation for both Newtonian and power law fluid with a 1D flow closed form solutions. Using geometry simplifications and an assemblage of mold sections, the total filling time, the inlet pressure or flow rate was calculated. The calculation showed a good agreement with Coulter and Güceri's TGMOLD (5). Methods based on 2D finite difference have been used by $\mathrm{Li}$ and Gauvin $(6)$ and by Coulter et al. $(7,8)$. The authors themselves notice the difficulty of finding realistic boundary conditions and point out the limitation of these methods for RTM. Finite element methods have been used by many authors as Trochu et al. (9) or Hoareau (10). The control volume method has been used by Lee et al. (11). These methods have the disadvantage of needing the meshing of either the whole preform or of the wetted region of the preform. Many authors have chosen a 2D formulation because the thickness of the component for impregnation is small in comparison with other dimensions. Nevertheless, Young et al. (12) have developed a 3D formulation of the finite element method with control volume method for thick components. With this coupled technique, remeshing at each time step is avoided and only the domain of calculation is recalculated at each time step.

In this study, following Um and Lee (13) and Hadavinia et al. (14), the resin flow will be solved numerically by the boundary element method (BEM). Traditional boundary element methods are particulary suitable for solving linear problems (Newtonian fluids). However, the methods have also been extented to solve nonlinear problems by iterative or incremental procedures. The variation of the boundary element method for a wide range of nonlinear problems including non-Newtonian fluids flows have been described in detail elsewhere $(15,16)$, and here we will only describe the technique when the resin is considered Newtonian. The results of numerical calculation will be compared to numerical solutions available for centrally injected anisotropic porous media. The numerical simulations will also be compared to finite elements results and experiments.

\section{GOVERNIG EgUATIONS}

The impregnation of a fibrous preform is usually modeled as a flow through anisotropic homogeneous porous media and is governed by Darcy's law:

$$
\vec{v}=-\frac{[K]}{\mu} \vec{\nabla} p
$$

where $\vec{v}$ is the velocity vector, $[K]=\left[\begin{array}{ll}K_{11} & K_{12} \\ K_{12} & K_{22}\end{array}\right]$ the permeability tensor, $p$ the resin pressure and $\mu$ the viscosity (constant for a Newtonian resin). Combining $E q 1$ with the continuity equation gives:

$$
\vec{\nabla} \cdot\left(-\frac{[K]}{\mu} \vec{\nabla} p\right)=0
$$

Equation 2 can be reduced to Laplace's equation by rotating and stretching the coordinates. The transformed coordinates $\vec{x}$ may be deduced from the system coordinates $\vec{X}$ using the following relationship:

$$
\vec{x}=\sqrt{K_{e}}\left[\begin{array}{cc}
\frac{\alpha_{1}}{\sqrt{\lambda_{1}}} & \frac{\beta_{1}}{\sqrt{\lambda_{1}}} \\
\frac{\alpha_{2}}{\sqrt{\lambda_{2}}} & \frac{\beta_{2}}{\sqrt{\lambda_{2}}}
\end{array}\right] \vec{X}
$$

where $\left(\alpha_{1}, \beta_{1}, \alpha_{2}, \beta_{2}\right)$ and $\left(\lambda_{1}, \lambda_{2}\right)$ are the components of the eigenvectors and the eigenvalues of the permeability matrix, respectively, and $K_{e}=\sqrt{\lambda_{1} \cdot \lambda_{2}}$ the equivalent permeability. Using the coordinates $\vec{x}$, Eqs 1 and 2 can be rewritten as:

$$
\begin{gathered}
\vec{v}=-\frac{K_{e}}{\mu} \vec{\nabla} p \\
\Delta p=0
\end{gathered}
$$

\subsection{Calculation of an Annhtical Solution}

Let us consider the radial impregnation of an anisotropic fibrous medium by an incompressible Newtonian fluid. Equations 4 and 5 are available. Using cylindrical coordinates, in $2 \mathrm{D}$ relative to the principal directions, the pressure can be calculated from $E q$ 5:

$$
\frac{p-p_{f}}{p_{0}-p_{f}}=1-\frac{\ln \left(R / R_{0}\right)}{\ln \left(R_{f} / R_{0}\right)}
$$

where $p_{0}$ and $p_{f}$ represent the inlet and front pressure respectively whereas $R_{0}$ and $R_{f}$ are the inlet and front radii. Fluid velocity, in the new system of coordinates, can be obtained from (4):

$$
v=\frac{K_{e}}{\mu} \frac{\left(p_{0}-p_{f}\right)}{R \ln \left(R_{f} / R_{0}\right)}
$$

The flow front velocity is given by the relationship between the phase (fluid) average $v$ to the intrinsic phase average $V$ (front velocity) (17).

$$
V_{f}=\frac{d R_{f}}{d t}=\frac{K_{e}}{\varepsilon \mu} \frac{\left(p_{0}-p_{f}\right)}{R_{f} \ln \left(R_{f} / R_{0}\right)}
$$

where $\varepsilon$ is the system porosity. If we assume that the porous medium is anisotropic and homogeneous. the 


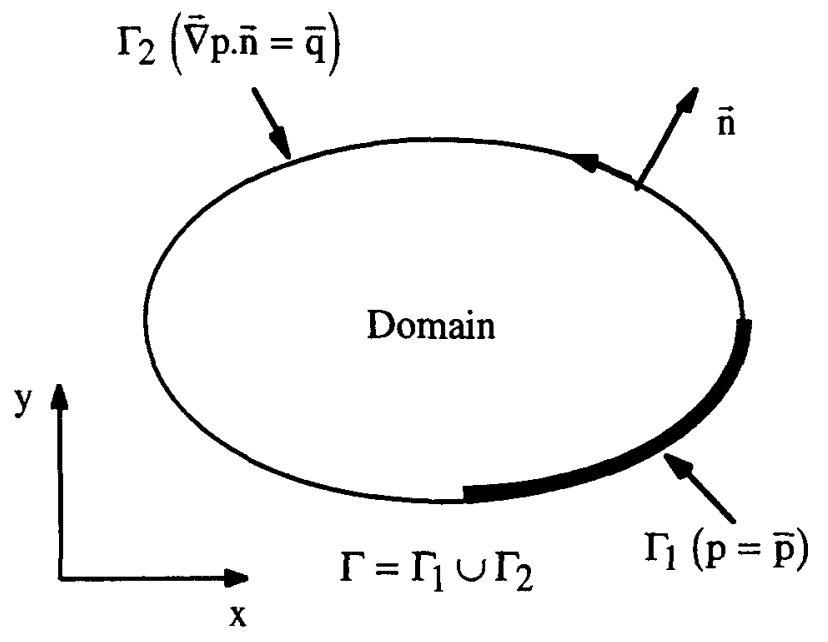

Fig. 1. Boundary conditions.

flow front should be of elliptical in shape. Hammami et al. (18) gave the following solution (for $P_{f}=0$ ):

$$
\left[\frac{R_{f}}{R_{0}}\right]^{2} \cdot\left[2 \ln \left(\frac{R_{f}}{R_{0}}\right)-1\right]+1=\frac{4 K_{e} p_{0} t}{\varepsilon \mu R_{0_{e}}^{2}}
$$

where $t$ is the elapsed time and $R_{0 e}$ the inlet radius in the transform domain.

\section{NUWTRICAL METEOD}

\subsection{Boundary Integral Equation}

The boundary conditions are sketched in Fig. $1 . \Gamma_{1}$ is the section of the boundary $\Gamma$ where a known value of pressure $\bar{p}$ is applied; $\Gamma_{2}$ the section of the boundary $\Gamma$ where a known value of pressure gradient $\bar{q}$ perpendicular to the solid wall is prescribed (for a non-penetration condition $\bar{q}=0$ ).

The transformed Eq 5 is multiplied by the weighting function $p^{*}$ and integrated over the calculation domain using Green's theorem to yield (19):

$$
c_{i} p_{i}+\int_{\Gamma} p q * d \Gamma=\int_{\Gamma} p^{*} q d \Gamma \text { with } \quad c_{i}=\frac{\theta}{2 \pi}
$$

where $p_{i}$ is the value of the pressure at a point $M_{i}$ located on the boundary (which is supposed to be smooth), $q^{*}$ the pressure gradient associated with $p^{*}$ and $\theta$ is the internal angle of the corner in radians. The weighting function $p^{*}$ is the fundamental solution satisfying:

$$
\Delta p^{*}+\delta_{i}=0
$$

where $\delta_{i}$ is the Dirac delta function. For a bidimensional medium, $p^{*}$ and $q^{*}$ are given as (20):

$$
p^{*}=\frac{1}{2 \pi} \ln \left(\frac{1}{r}\right) \text { and } q^{*}=\frac{1}{2 \pi} \frac{-\vec{r} \cdot \vec{n}}{r^{2}}
$$

where $r$ is the distance from the point $M_{i}$ of application of the Dirac delta function to any point under consideration.
Table 1. Processing and Rheological Parameters.

\begin{tabular}{cccc}
\hline$[\mathrm{K}]\left(10^{-11} \mathrm{~m}^{2}\right)$ & $\mu$ (Pa.s) & $\mathrm{p}_{0}(\mathrm{~Pa})$ & $\mathrm{R}_{0}(\mathrm{~m})$ \\
\hline$\left[\begin{array}{cc}4 & 2.5 \\
2.5 & 4\end{array}\right]$ & 0.1 & $10^{5}$ & 0.001 \\
\hline
\end{tabular}

\subsection{Boundary Element Equation}

Elements used are linear and the variation of $p$ and $q$ for a given element $\Gamma_{j}$ are defined as:

$$
\begin{gathered}
p_{j}(\xi)=\sum_{i=1}^{2} \Phi_{i}(\xi) p_{j}^{l} \quad \Phi_{1}(\xi)=\frac{1}{2}(1-\xi) \\
\text { where } \\
q_{j}(\xi)=\sum_{i=1}^{2} \Phi_{i}(\xi) q_{j}^{l} \quad \Phi_{2}(\xi)=\frac{1}{2}(1+\xi)
\end{gathered}
$$

where $\xi$ is the dimensionless coordinate $[-1 ; 1]$. Dividing the boundary into $N$ linear boundary elements and applying $E q 10$ :

$$
c_{i} p_{i}+\sum_{j=1}^{N}\left[h_{i j}^{1} h_{i j}^{2}\right]\left\{\begin{array}{l}
p_{j}^{1} \\
p_{j}^{2}
\end{array}\right\}=\sum_{j=1}^{N}\left[\begin{array}{ll}
g_{i j}^{1} & g_{i j}^{2}
\end{array}\right]\left\{\begin{array}{l}
q_{j}^{1} \\
q_{j}^{2}
\end{array}\right\}
$$

where

$$
\begin{aligned}
& h_{i j}^{k}=\int_{\Gamma_{j}} \Phi_{k}(\xi) q^{*} d \Gamma \\
& g_{i j}^{k}=\int_{\Gamma_{J}} \Phi_{k}(\xi) p^{*} d \Gamma
\end{aligned}
$$

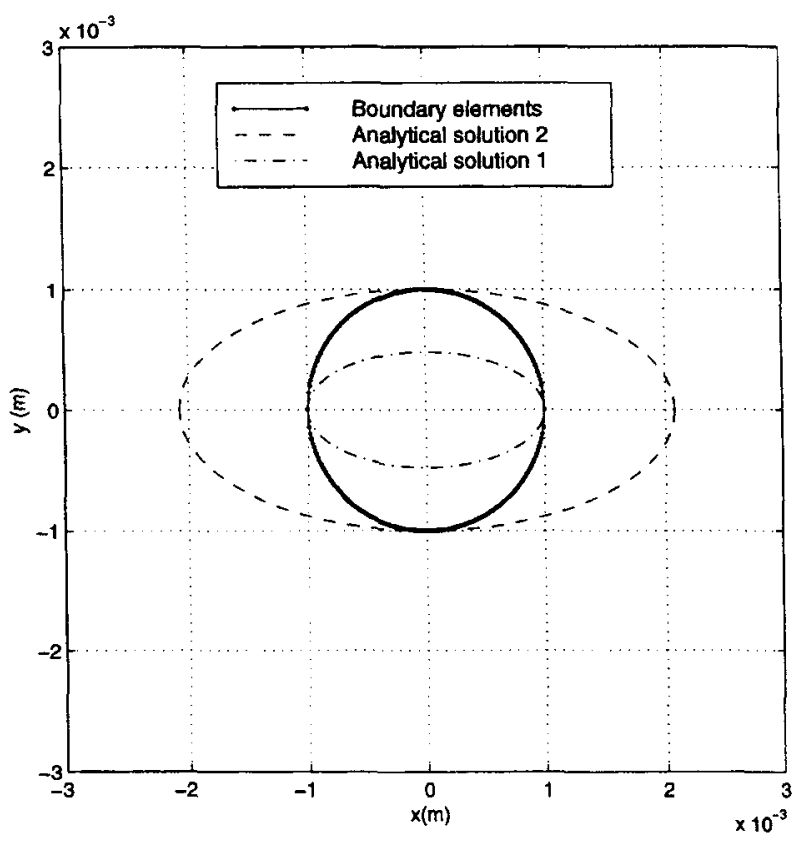

Fig. 2. Circular inlet between two ellipses. 
Since pressure is unique at any point:

$$
\forall j \in[2 ; N] p_{j-1}^{2}=p_{j}^{1} \text { and } p_{N}^{2}=p_{N+1}^{1}=p_{1}^{1}
$$

Equation 14 can be rewritten in matrix form as:

$$
\left[\begin{array}{llll}
H_{i 1} & H_{i 2} & \cdots & H_{i N}
\end{array}\right]\left\{\begin{array}{c}
p_{1} \\
p_{2} \\
\vdots \\
p_{N}
\end{array}\right\}=\left[\begin{array}{llll}
G_{i 1} & G_{i 2} & \cdots & G_{i 2 N}
\end{array}\right]\left\{\begin{array}{c}
q_{1} \\
q_{2} \\
\vdots \\
q_{2 N}
\end{array}\right\}
$$

where:

$$
H_{i j}=h_{i j-1}^{2}+h_{i j}^{1}+\delta_{i j} c_{i}
$$

Fluxes are unique at any point of smooth surfaces and unknown after or before a corner (transition from known pressure to known flux or vice versa). As long as there is only one unknown per node, a $N^{*} N$ system can be obtained. Such a system can be reordered as:

$$
A X=F
$$

where $X$ is the vector of $N$ unknowns, $A$ is a $N^{*} N$ matrix obtained by reordering $G$ and $H . F$ is the known vector computed from the boundary conditions and the $H$ and $G$ matrices.

\subsection{Resin Front Updating}

At each time step $\Delta t$, the flow of resin inside the mold is regarded as quasi-steady. Unknown pressure $p$ and pressure gradient $q$ on the boundary are calculated by using $E q 17$. Then, the new location of the resin front is updated using a forward Euler integration scheme:

$$
\vec{x}(t+\Delta t)=\vec{x}(t)+\Delta t(\vec{v} \cdot \vec{n}) \vec{n}
$$

On the resin front, only the normal component of the resin velocity is needed since there is no tangential component of the velocity. Combining Eqs 4 and 18 we obtain:

$$
\vec{x}(t+\Delta t)=\vec{x}(t)-\Delta t\left(K_{e} \frac{q}{\mu} \vec{n}\right)
$$

In addition, the nodes close to the mold are relocated using an orthogonal condition.

\section{MODEL AGSESSMMNT}

The boundary elements method developed for the RTM process was compared to analytical solutions, finite element simulations and experimental data. The first case considered was the central injection of an anisotropic and orthotropic fibrous medium. The porous media was rotated with an angle of $45^{\circ}$ for a purpose of generality. The values of the different parameters used are given in Table 1.

The matrix $[K]$ is the permeability matrix in the $\vec{X}$ coordinates system. It is the expression of the principal permeability matrix $\left[\begin{array}{cc}6,5.10^{-11} & 0 \\ 0 & 1,5.10^{-11}\end{array}\right]$ relative to coordinates such as $(\vec{X}, \vec{x})=45^{\circ}$. The flow front should be of elliptical shape. The half-lengths $R_{f x p}$ and
Fig. 3. Comparison between BEM and analytical flow fronts $(t=$ $200 \mathrm{~s})$.

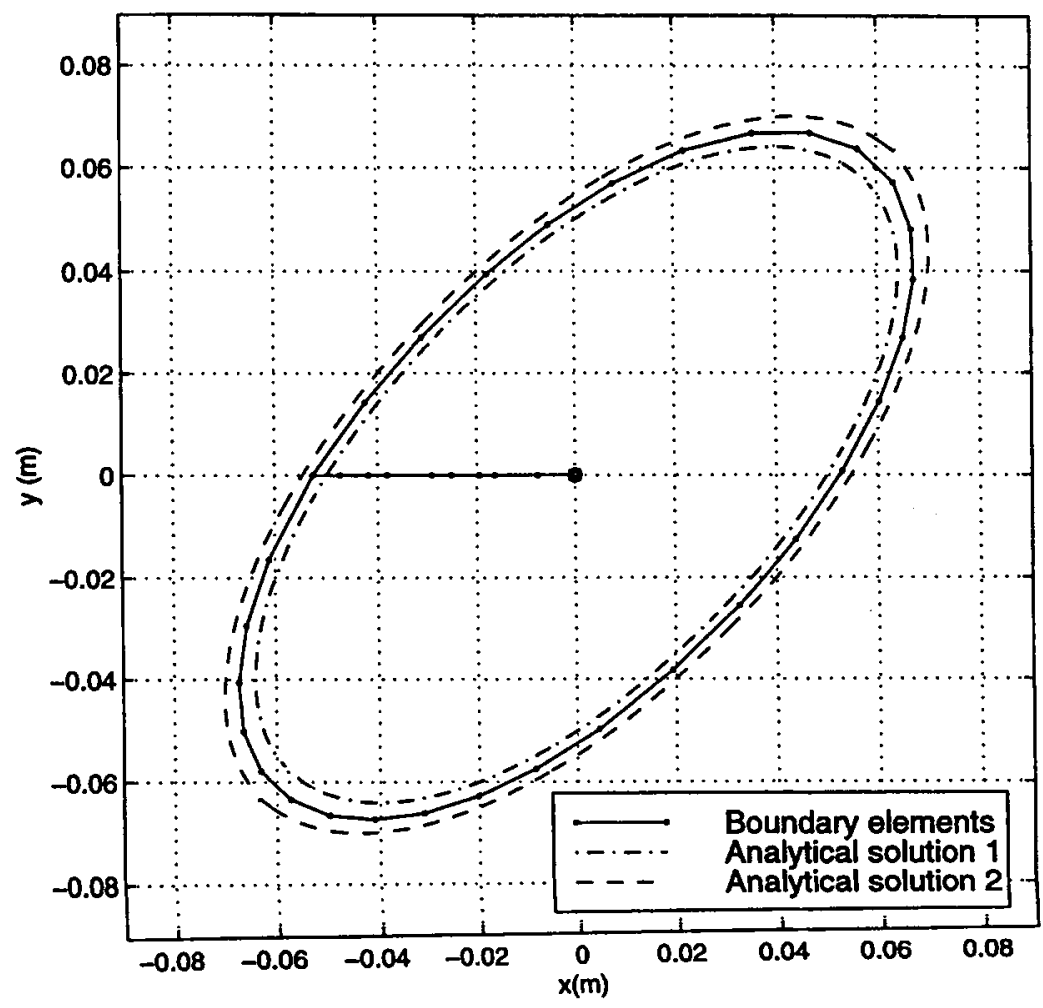


Fig. 4. Comparison between linear and contant BEM and analytical flow fronts.

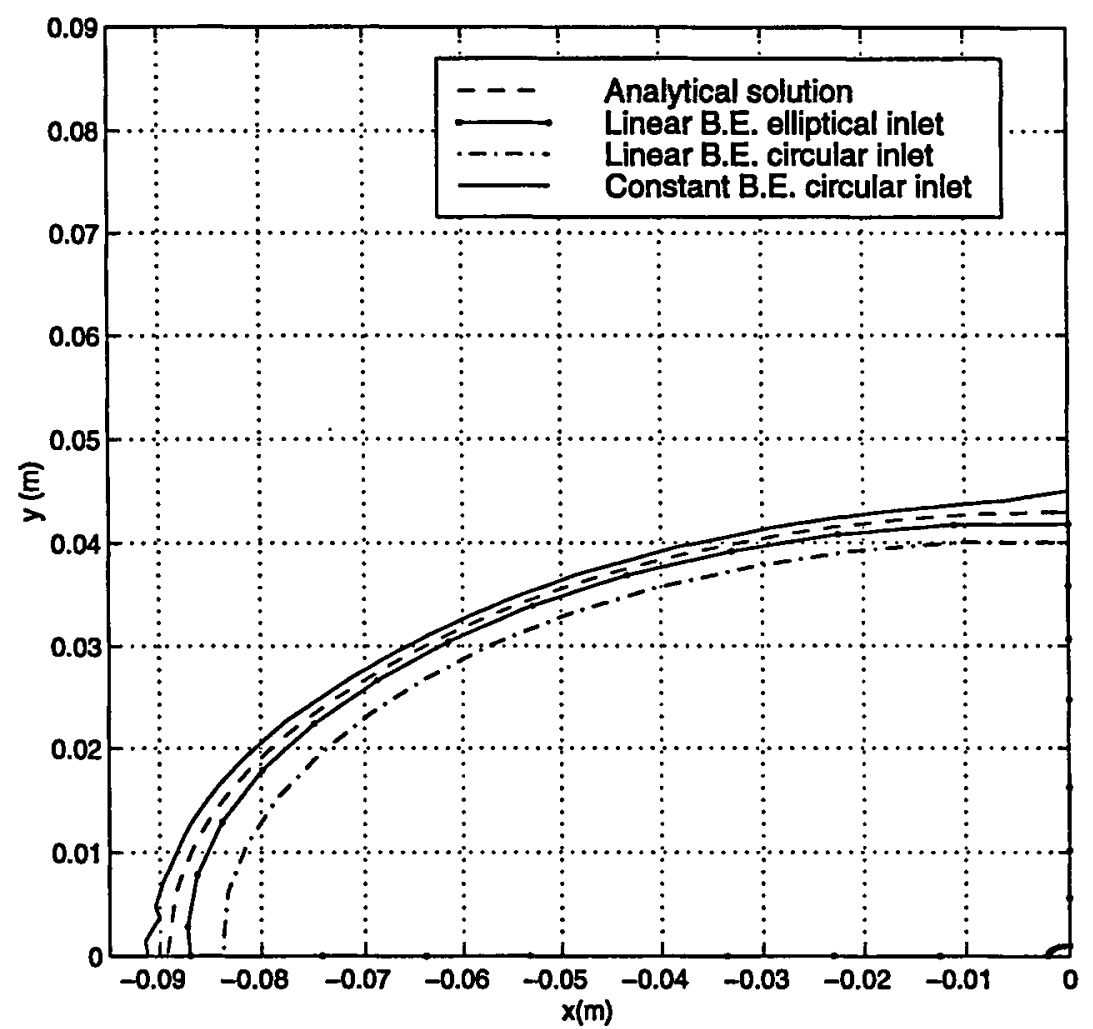

Table 2. Processing and Rheological Parameters.

\begin{tabular}{cccc}
\hline$[\mathrm{K}]\left(10^{-11} \mathrm{~m}^{2}\right)$ & $\mu$ (Pa.s) & $p_{0}(\mathrm{~Pa})$ & $R_{0}(\mathrm{~m})$ \\
\hline$\left[\begin{array}{cc}6.5 & 0 \\
0 & 1.5\end{array}\right]$ & 0.1 & $10^{5}$ & 0.001 \\
\hline
\end{tabular}

$R_{f y p}$ of the principal axis of the ellipse can be computed with $E q$ 9. The half-lengths of ellipses are related to principal permeabilities by:

$$
\frac{R_{x}}{R_{y}}=\sqrt{\frac{\lambda_{1}}{\lambda_{2}}}=\sqrt{\frac{6,5}{1,5}}=2,08
$$

In $E q$ 9, the pressure $p_{0}$ is applied in the $\vec{X}$ system coordinates which implies an elliptical inlet. In fact, there is two possible elliptical inlets. If we proceed to a BEM simulation with a circular inlet located between these two ellipses (see Fig. 2), the BEM flow front should be located between the analytical flow fronts corresponding to these ellipses.

Figure 3 shows the comparison between the flow front which has been computed using the boundary element method with linear elements and the analytical solutions at $t=200 \mathrm{~s}$ for ellipses of half axes $(0,00208 ; 0,001)$ and $(0,001 ; 0,00048)$. The time step used for the numerical simulation is $\Delta t=0.1 \mathrm{~s}$ and the CPU time for the complete simulation is one minute on IBM Risk 6000. The simulation is in good agreement with the analytical solution and the calculated flow front is located between the analytical solu- tions corresponding to the half-axes. Figure 3 also shows the sensitivity of the method to the selected inlet geometry. A small variation of the inlet geometry has important role on the shape of the flow front position.

In Fig. 4, different boundary element simulations are shown for an orthotropic case for one quarter of the domain. The values of the processing and rheological parameters are given in Table 2.

The boundary element simulation with constant elements leads to some problems close to the mold wall (Fig. 4). Moreover, it overestimates the flow front position. Linear elements improve the contact description. Two different inlet geometry were compared; a circular inlet and an elliptical one. The difference between the boundary element method and the analytical solutions can be explained by the sensitivity of the inlet geometry.

\subsection{Comparison With Pinite Blement Simulation}

A second system consisting of a frontal injection of resin into a fibrous preform located between two parallel solid walls was used to compare finite element and boundary element methods. The resin was assumed to be an incompressible Newtonian fluid flowing through an anisotropic one layer medium.

The finite element simulation has been carried out using a numerical model first developed by Hoareau et al. (21) and derived from FORGE2 ${ }^{\circledR}$, software. The numerical model is based upon an updated lagrangian finite element method (FEM). The domain is meshed using quadratic triangles (six nodes) as shown in Fig. 


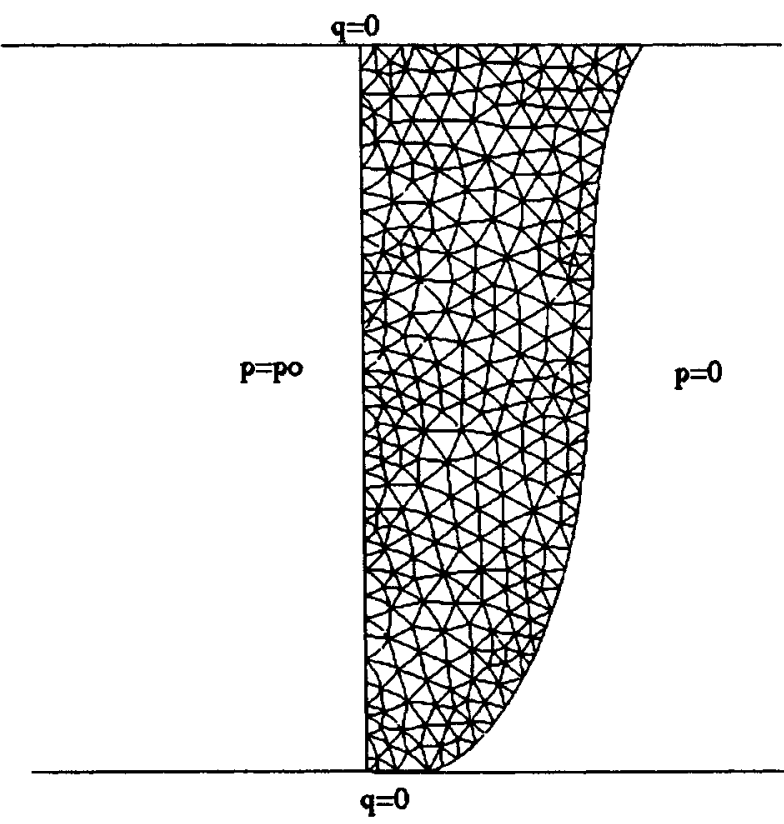

Fig. 5. Finite element mesh at $t=200 \mathrm{~s}$.

5. The values of the processing and rheological parameters are the same as the one given in Table 1 . The CPU time for the FEM simulation is about halfan-hour. As shown in Fig. 6, the agreement is fair for both constant and linear boundary elements except close to the lower wall. As expected, we have a better agreement for linear elements. The discrepancy between the BEM and FEM flow fronts increases in the lower part.

\subsection{Comparison With Experimental Data}

Experimental data available for the case of central injection into a transparent mold was used to compare BEM and experimental measurement. The dimensions of the mold are $0.5 \mathrm{~m}$ per $1 \mathrm{~m} \mathrm{(Fig.} \mathrm{7)} \mathrm{and}$ a silicone oil resin was used to impregnate an isotropic fibrous medium made of six layers of OCF 8610 fiber mat. All details of the procedure can be found in reference (22). The values of the processing and rheological parameters are given in Table 3 . The CPU time for the complete simulation is $2 \mathrm{~min}$. The agreement between the BEM and the experimental data is fair except near the walls. The numerical flow fronts shown in Fig. 8.a are symmetric while the experimental ones are non-symmetric (Fig. 8.b). This is due to the difficulties of placing the preform of reinforcing fibers perfectly in contact with the mold walls. The permeability will increase near the wall generating preferential flows.

\section{5 conclusion}

The flow of a Newtonian resin through anisotropic and homogeneous media has been simulated using a boundary element method. The algorithm which is used to capture the transient front flow is rather simple and can accurately predict the front shape at a
Fig. 6. Comparison between BEM and FEM flow fronts at $t=200 \mathrm{~s}$.

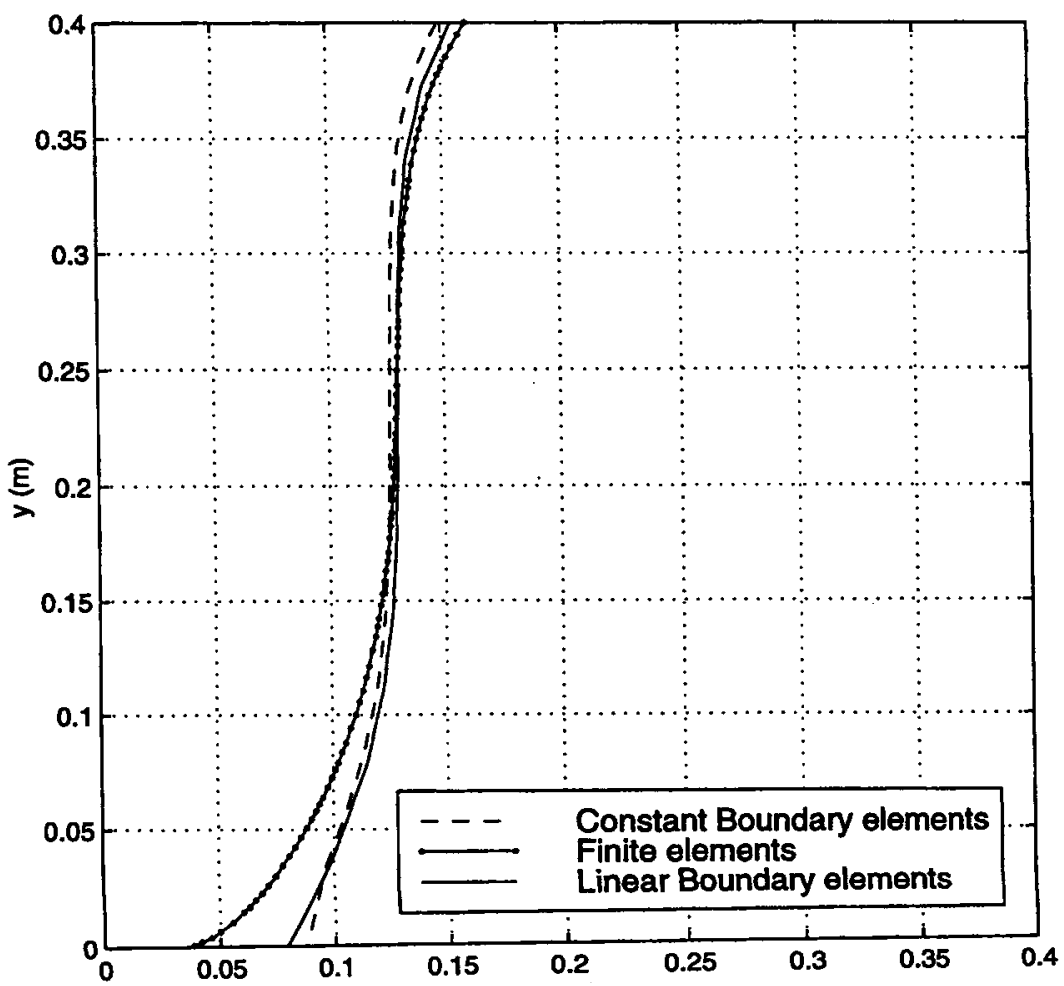


Fig. 7. Cross section of the experimental mold-h thickness of the mold.

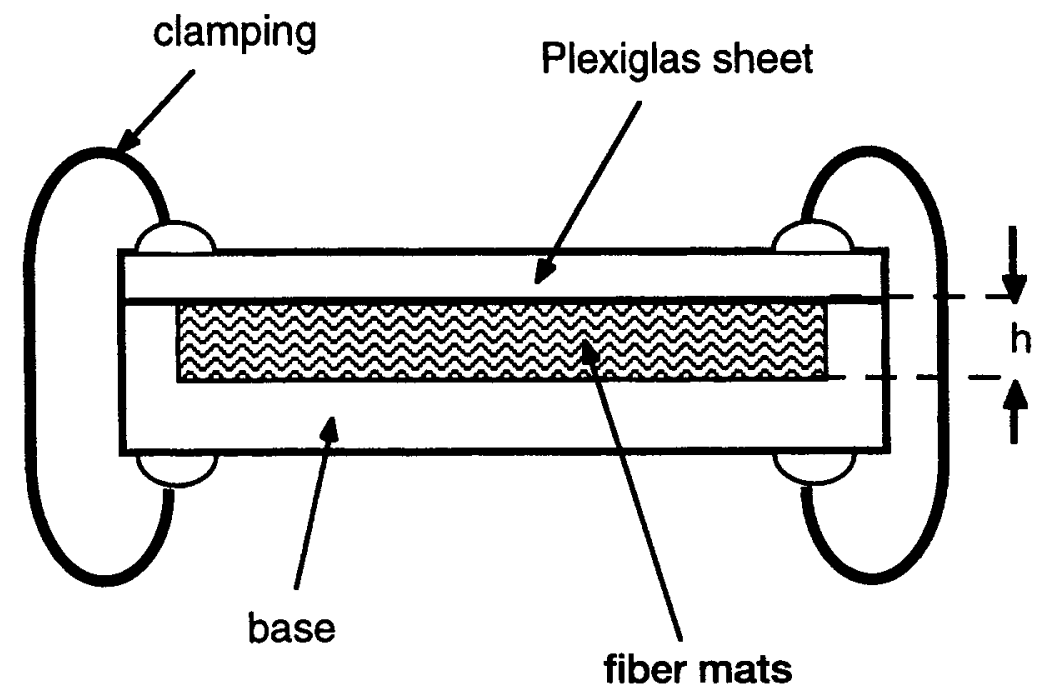

Table 3. Processing and Rheological Parameters.

\begin{tabular}{cccc}
\hline$[K]\left(10^{-8} \mathrm{~m}^{2}\right)$ & $\mu$ (Pa.s) & $p_{0}(\mathrm{~Pa})$ & $R_{0}(\mathrm{~m})$ \\
\hline$\left[\begin{array}{cc}1.7 & 0 \\
0 & 1.7\end{array}\right]$ & 0.082 & 15 & 0.01 \\
\hline
\end{tabular}

low cost. The numerical model has been validated by comparison with analytical solutions for a central injection and with finite element for a frontal injection. The anisotropic and orthotropic fibrous medium have been investigated.
Fig. 8. Comparison between BEM simulations (a) and experiments (b).

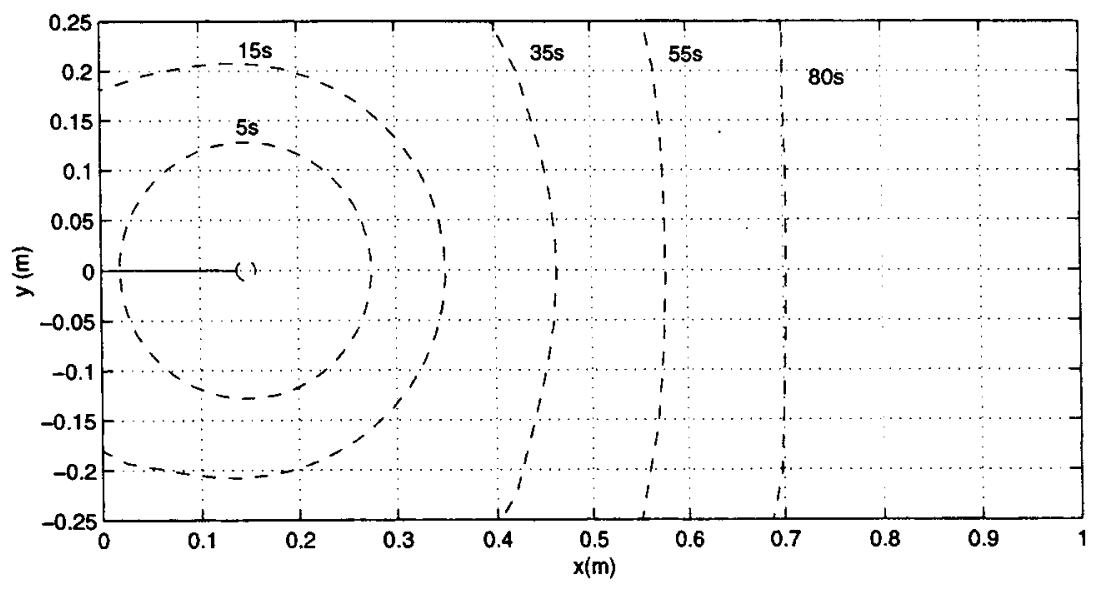

(a)

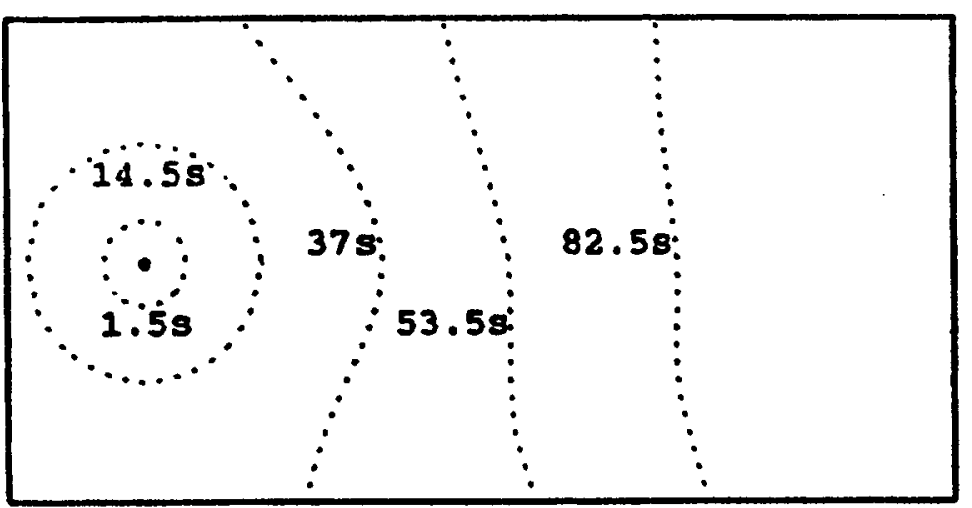


Preliminary comparison with experimental data available for the case of central injection into a transparent mould have been processed. However, further comparisons between BEM simulations and experiments are needed to improve the model. In particular, the relocation scheme used for the nodes close to the mold has to be improved in order to take into account the contact between the resin and the mold.

Further developments will allow the simulation of the flow of resin through a typical RTM reinforcement (i.e. inhomogeneous porous media). The boundary element method will be applied to each subregion (assumed to be piecewise homogenous) as if they were independent of each other.

\section{REFERENCES}

1. C. F. Johnson, N. G. Chavka, R. A. Jeryan, C. J. Morris, and D. A. Balbington, Design and fabrication of a HSRTM crossmember module, proceedings of the third annual ASM/ESD advanced Composites Conference, pp. 197-217 (1987).

2. A. Arnold and W. Becker, RTM: simultaneous design and tooling reduces cost and lead time, Proceedings of the 23rd International SAMPE Technical Conferences (1991).

3. D. A. Steenkamer, The influence of preform design and manufacturing issues on the processing and performance of resin transfer molded composites (Volume I and II). Thesis of University of Delaware (1994).

4. Z. Cai, Simplified mold filling simulation in resin transfer molding, Journal of Composite Materials, Vol. 26, $\mathrm{N}^{\circ}$ 17, p. 2606-2630 (1992).

5. J. P. Coulter and S. I. Guceri, resin transfer molding: process review, modeling and research opportunitiesProceedings of Manufacturing International 88, Atlanta GA, No4, p. 79, 86 (1988).

6. S. Li and R. Gauvin, Numerical Analysis of the Resin Flow in Resin Transfer Molding. Journal of Reinforced Plastics and Composites, Vol. 10, 314-327 (May 1991).

7. J. P. Coulter and S. 1. Guceri, resin impregnation during the manufacturing of composite materials subject to prescribed injection rate- Joumal of reinforced plastics and composites, May, Vol. 7, p. 200-2 19 (1988).

8. J. P. Coulter and S. I. Guceri, resin impregnation during composites manufacturing: theory and experimentation, Composites Science and Technology, Vol. 35, p. 317-330 (1989).
9. F. Trochu, R. Gauvin, Gao Dong Ming, and J.-F. Boudreault, RTMFLOT, An Integrated Software Environment for the Computer Simulation of the Resin Transfer Molding Process. Journal of Reinforced Plastics and Composites, Vol. 13, 262-270 (March 1994).

10. C. Hoareau, F. Trochu, R. Gauvin, and M. Vincent, Proc. of ICCM-9, Madrid, Vol. 3, pp. 481-488 (July 1993).

11. L. James Lee, W. B. Young, and R. J. Lin, Mold filling and cure modelling of RTM and SRIM processes. Composites Structures, Vol. 27, 109-120 (1994).

12. W. B. Young, K. H. L. M. Fong, L. J. Lee, and M. J. Liou, Flow simulation in molds with preplaced fiber mats, Polymer Composites, 12, p. 391 (1991).

13. M.-K. Um and W. I. Lee, A study on the Mold Filling Process In the Resin Transfer Molding. Polymer Engineering and Science, Vol. 31, No. 11, 765-771 (MidJune, 1991).

14. H. Hadavinia, S. G. Advani, and R. T. Fenner, The evolution of radial fingering in a hele-Shaw cell using $\mathrm{C}^{\mathrm{l}}$ continuous Overhauser boundary element method. Engineering Analysis Boundary Elements, Vol. 16, 183195 (1995).

15. M. B. Bush. The application of Boundary Element Method to Some Fluid Mechanics Problems, PhD Thesis, University of Sydney (1984).

16. R. Zheng. Boundary Element Method for some problems in Fluid Mechanics and Rheology, PhD Thesis, University of Sydney (1991).

17. C. L. Tucker, III, and R. B. Dessenberger, "Chapter 8: Governing equations for flow and heat transfer in stationary fiber beds," Flow and rheology in polymer composites manufacturing edited by $\mathbf{S}$. G. Advani, Elsevier Science B. V. (1994).

18. A. Hammami, F. Trochu, R. Gauvin \& S. Wirth, Directional Permeability Measurement of Deformed Reinforcement. J. of Reinforced Plastics and Composites, Vol. 15 (June 1996).

19. C. A. Brebbia and J. Dominguez, Boundary Element: An introduction course. McGraw-Hill Book Company, Second edition (1992).

20. N. Ozisik, Heat Conduction, John Wiley \& Sons (1980).

21. C. Hoareau, Injection sur renfort: etude du remplissage de moule et détermination théorique de la perméabilité des tissus, thèse de doctorat de l'école des mines de Paris, in French (1994).

22. Zhang, Zheng, * Simulation par éléments finis du remplissage des moules par le procédé de moulage par transfert de résine," Mémoire de Maitrise, in French. École Poytechnique de Montréal (1992). 